$N D B$

$1 N-27-7 M$

\title{
Internal Radiation Effects in Zirconia Thermal Barrier Coatings R. Siegel
}

Reprinted from

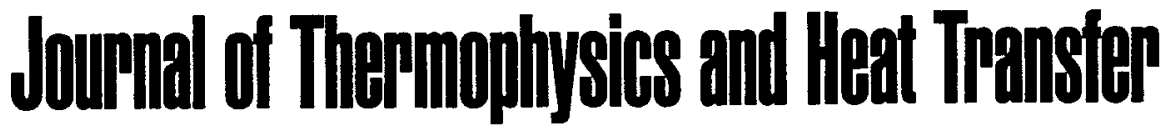

Volume 10, Number 4, Pages 707-709

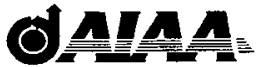

A publication of the

American Institute of Aeronautics and Astronautics, Inc.

1801 Alexander Bell Drive, Suite 500

Reston, VA 22091 


\section{Internal Radiation Effects in Zirconia Thermal Barrier Coatings}

\author{
Robert Siegel* \\ NASA Lewis Research Center, Cleveland, Ohio 44135
}

\section{Nomenclature}

$a=$ absorption coefficient of semitransparent coating, $\mathrm{m}^{-1}$

$c_{0}=$ speed of electromagnetic propagation in vacuum, $\mathrm{m} / \mathrm{s}$

$G \quad=$ flux quantity $2\left(q_{r}^{+}+q_{r}^{-}\right), \mathrm{W} / \mathrm{m}^{2} ; G_{L}=G / \sigma T_{s !}^{4}$ in large-frequency spectral band

$h_{1}, h_{2}=$ convective heat transfer coefficients at boundaries, $\mathrm{W} / \mathrm{m}^{2} \mathrm{~K} ; H=h / \sigma T_{s 1}^{3}$

$K=$ extinction coefficient of semitransparent material, $a+\sigma_{r}, \mathrm{~m}^{-1}$

$k_{c}, k_{m}=$ thermal conductivity (coating, metal), W/mK; $N_{c}=k_{c} / \sigma T_{x_{1}}^{3} \delta_{c}$

$n \quad=$ refractive index of semitransparent material

$q \quad=$ heat flux, $\mathrm{W} / \mathrm{m}^{2} ; q_{r}=$ radiative flux, $\mathrm{W} / \mathrm{m}^{2}$; $\tilde{q}=q / \sigma T_{g 1}^{4}$

$q_{r}^{+}, q_{r}^{-}=$isotropic radiative fluxes in $+x$ and $-x$ directions, Fig. $1, \mathrm{~W} / \mathrm{m}^{2}$

$T \quad=$ absolute temperature, $\mathrm{K} ; t=T / T_{\text {it }}$

$T_{\xi 1}, T_{\xi 2}=$ gas temperatures on hot and cold sides of composite, Fig. 1, K

$T_{s 1}, T_{s 2}=$ temperatures of surroundings on two sides of composite, Fig. 1, K

$x=$ coordinate in composite, $\mathrm{m} ; X=x / \delta_{c}$

$\delta_{c}, \delta_{u}=$ thicknesses of coating and metal, $\mathrm{m}$

$\varepsilon \quad=$ emissivity

$\kappa_{c L} \quad=$ optical thickness of coating in large-frequency band, $K_{L} \delta_{c}$

$\lambda, \nu \quad=$ wavelength and frequency of radiation; $\nu_{\mathrm{co}}=$ cutoff frequency

$\rho=\quad=$ diffuse reflectivity of interface from Fresnel equations

$\sigma \quad=$ Stefan - Boltzmann constant, $\mathrm{W} / \mathrm{m}^{2} \mathrm{~K}^{4}$

$\sigma_{s} \quad=$ scattering coefficient in semitransparent coating, $\mathrm{m}^{-1} ; \Omega=\sigma_{s} / K$

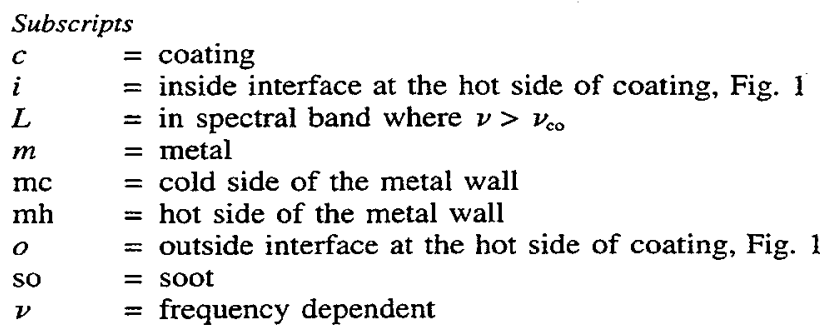

\section{Introduction}

T SING thermal barrier coatings on combustor liners, turbine vanes, and rotating blades is important for reducing metal temperatures in current and advanced aircraft engines. Zirconia is a common coating material, and it is partially transparent to thermal radiation. Radiation becomes more significant as temperatures are raised for higher efficiency in advanced engines. Calculations are often made with radiation effects neglected inside the coating. The effect of radiation is illustrated here, where an analytical procedure is provided by using the two-flux method for the radiative contribution.

In Ref. 1 a detailed study was made of ceramic thermal barrier coatings for diesel engines. In Ref. 2 a two-flux analysis was developed for radiation in semitransparent multilayer composites. These references provide the basis for the present analysis where illustrative solutions are obtained for typical conditions in an aircraft engine. The formulation and solution of the exact spectral radiative transfer equations including large scattering, as is characteristic of zirconia, are rather complicated. For plane-layer composites, the two-flux equations were shown in Ref. 2 to give accurate results for gray and spectral layers; hence, the two-flux method is used here to provide a simplified method.

\section{Analysis}

A composite is first considered of a thermal barrier coating on a metal wall with the external surface of the coating covered with a thin opaque layer of soot (upper part of Fig. 1). For an advanced engine, the combustion chamber pressure is

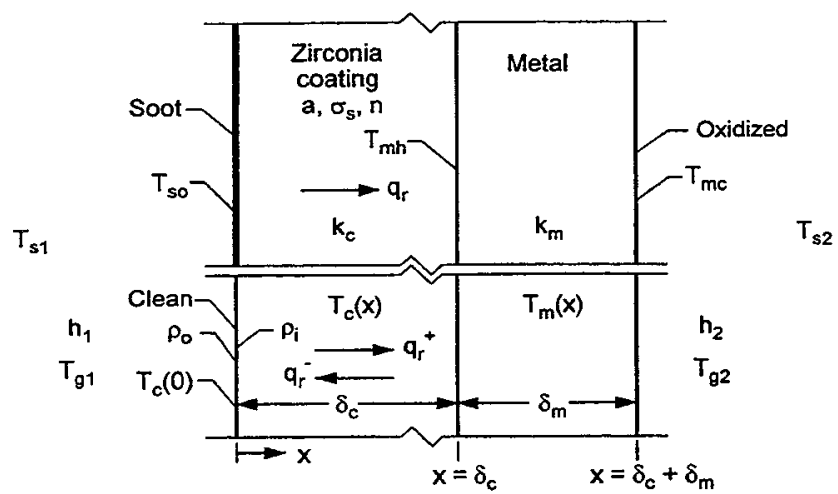

Fig. 1 Geometry and nomenclature for a thermal barrier coating on a metal wall with and without soot on the exposed surface of the coating.
Received May 16, 1996; revision received June 7, 1996; accepted for publication June 9, 1996. Copyright (C) 1996 by the American Institute of Aeronautics and Astronautics, Inc. No copyright is asserted in the United States under Title 17, U.S. Code. The U.S. Government has a royalty-free license to exercise all rights under the copyright claimed herein for Governmental purposes. All other rights are reserved by the copyright owner.

* Senior Research Scientist, Research Academy, 21000 Brookpark Road. Fellow AIAA. 
high enough that the combined gas and soot radiation can be assumed to provide a black environment at $T_{s 1}$. At the outer surface of the soot layer, the total heat flux consists of convection and radiation,

$$
q_{\mathrm{tot}}=h_{1}\left(T_{\xi 1}-T_{\mathrm{so}}\right)+\varepsilon_{\mathrm{so}} \sigma\left(T_{s 1}^{4}-T_{\mathrm{so}}^{4}\right)
$$

The soot layer is very thin, and so temperature variation through its thickness is neglected. Within the semitransparent thermal barrier coating, the total heat flux is the sum of conduction and radiation,

$$
q_{\mathrm{tot}}=-k_{c} \frac{\mathrm{d} T_{c}(x)}{\mathrm{d} x}+\int_{\nu \neq 0}^{\infty} q_{\nu r}(x) \mathrm{d} \nu
$$

The $q_{\nu r}$ is eliminated by using the two-flux equation ${ }^{3}$ (1/ $\left.K_{\nu}\right)\left(\mathrm{d} G_{\nu} / \mathrm{d} x\right) \mathrm{d} \nu=-3 q_{\nu r}(x) \mathrm{d} \nu$, and the result is integrated in the coating from $x=0$ to $x$, to yield the total heat flux as

$$
q_{\mathrm{tot}} x=k_{c}\left[T_{c}(0)-T_{c}(x)\right]+\frac{1}{3} \int_{\nu=0}^{\infty} \frac{G_{\nu}(0)-G_{\nu}(x)}{K_{\nu}} \mathrm{d} \nu
$$

The two-flux equation for $G_{\nu}$ is a second-order differential equation that contains the local blackbody emission, ${ }^{3} n^{2} e_{\nu b}[\nu$, $T(x)]$

$$
-\frac{1}{3 K_{\nu}^{2}} \frac{\mathrm{d}^{2} G_{\nu}(x)}{\mathrm{d} x^{2}} \mathrm{~d} \nu=\left(1-\Omega_{\nu}\right)\left[4 n^{2} e_{\nu b}(x) \mathrm{d} \nu-G_{\nu}(x) \mathrm{d} \nu\right]
$$

where $e_{\nu b}(\nu, T) \mathrm{d} \nu=2 \pi C_{1} \nu^{3} \mathrm{~d} \nu / c_{0}^{4}\left[\exp \left(C_{2} \nu / c_{0} T\right)-1\right],\left(C_{1}\right.$ and $C_{2}$ are the blackbody radiation constants). Two boundary conditions are required for solving Eq. (4). At the interface ( $x$ $=0$ ) of the soot and the semitransparent coating, the radiative flux within the coating in the positive direction is composed of emission and reflection by the soot, $q_{\nu r}^{+}(0)=\varepsilon_{\mathrm{so}} n^{2} e_{\nu b}(0)+$ $\left(1-\varepsilon_{\mathrm{so}}\right) q_{\nu r}^{-}(0)$. The two-flux relations, ${ }^{3} q_{\nu r}^{+}=(1 / 2)\left[\left(G_{\nu} / 2\right)+\right.$ $\left.q_{\nu r}\right]$ and $q_{\nu r}^{-}=(1 / 2)\left[\left(G_{\nu} / 2\right)-q_{\nu r}\right]$ are used to eliminate $q_{\nu r}^{+}$and $q_{v r}^{-}$, and the two-flux equation following Eq. (2) is used to eliminate $q_{\nu r}$ in terms of $\mathrm{d} G_{\nu} / \mathrm{d} x$. This yields the boundary condition for $G_{\nu}$ at $x=0$ :

$$
\left.\frac{\mathrm{d} G_{\nu}}{\mathrm{d} x}\right|_{x=0}=\frac{3 K_{\nu} \varepsilon_{\mathrm{so}}}{2\left(2-\varepsilon_{\mathrm{so}}\right)}\left[G_{\nu}(0)-4 n^{2} e_{\nu b}(0)\right]
$$

A similar derivation gives the boundary condition for $G_{\nu}$ at $x=\delta_{c}$ :

$$
\left.\frac{\mathrm{d} G_{\nu}}{\mathrm{d} x}\right|_{x=\delta_{c}}=-\frac{3 K_{\nu} \varepsilon_{\mathrm{mh}}}{2\left(2-\varepsilon_{\mathrm{mh}}\right)}\left[G_{\nu}\left(\delta_{c}\right)-4 n^{2} e_{\nu b}\left(\delta_{c}\right)\right]
$$

If there is no soot coating, so that the thermal barrier coating has a clean external surface (lower part of Fig. 1), the boundary condition for $G_{\nu}$ at the external boundary of the coating is $^{2,3}$

$$
G_{\nu}(0)=4 \frac{1-\rho_{o}}{1-\rho_{i}} e_{\nu b}\left(\nu, T_{s 1}\right)+\left.\frac{2}{3 K_{\nu}} \frac{1+\rho_{i}}{1-\rho_{i}} \frac{\mathrm{d} G_{\nu}}{\mathrm{d} x}\right|_{x=0}
$$

At the interface between the semitransparent coating and metal, continuity of temperature gives $T_{c}\left(\delta_{c}\right)=T_{m}\left(\delta_{c}\right) \equiv T_{\mathrm{mh}}$. For the opaque metal wall, heat is transferred only by conduction:

$$
q_{\mathrm{tot}}=k_{\mathrm{sn}}\left[\left(T_{\mathrm{mh}}-T_{\mathrm{mc}}\right) / \delta_{m}\right]
$$

At the cooled side of the metal wall, heat flow is by convection to the cooling gas, and by radiation to the surrounding structure. Since radiative transfer is appreciable, two limiting cases can be considered. For $\varepsilon_{i,}$, there is an adjacent metal wall at $T_{s 2}$, such that radiative loss is by transfer between parallel plates. For $\varepsilon_{x}$, large surroundings at $T_{s 2}$ are assumed:

$$
\begin{aligned}
& q_{\mathrm{tor}}=h_{2}\left(T_{\mathrm{rnc}}-T_{g^{2}}\right)+\left(\varepsilon_{\|} \text {or } \varepsilon_{x}\right) \sigma\left(T_{\mathrm{mc}}^{4}-T_{s 2}^{4}\right) \\
& \varepsilon_{\| t}=\varepsilon_{\mathrm{mc}} /\left(2-\varepsilon_{\mathrm{mc}}\right), \quad \varepsilon_{x}=\varepsilon_{\mathrm{mc}}
\end{aligned}
$$

Two-Band Form of the Radiative Relations

For a zirconia thermal barrier coating, the semitransparent region extends to a cutoff wavelength of about $5 \mu \mathrm{m}$, beyond which the extinction coefficient becomes quite large. ${ }^{1.5}$ From the spectral property variations of zirconia, a two-band approximation is reasonable, where the band for large wavelengths (small frequencies) is considered opaque.

To obtain the temperature distribution in the thermal barrier coating, Eq. (3) is integrated over the semitransparent band (large frequencies) and the result rearranged to give in dimensionless form

$$
t_{c}(X)=t_{c}(0)-\frac{1}{N_{c}}\left[\tilde{q}_{\mathrm{tot}} X-\frac{G_{L}(0)-G_{L}(X)}{3 \kappa_{c L}}\right]
$$

To obtain $G_{L}(X)$, Eq. (4) is solved in the form

$$
\frac{\mathrm{d}^{2} G_{L}(X)}{\mathrm{d} X^{2}}=-3 \kappa_{c L}^{2}\left(1-\Omega_{L}\right)\left\{4 n^{2} t_{c}(X)^{4} F\left[t_{c}(X)\right]-G_{L}(X)\right\}
$$

where $F(t)$ is the fraction of blackbody energy in the frequency range $\nu=\nu_{\mathrm{co}}-\infty$ for emission at $t ; F(t)$ was evaluated from the series form in Ref. 4, Chap. 2. For Eq. (9), there are two cases for boundary conditions: 1) an opaque soot coating on the zirconia, and 2) the zirconia surface is clean. For an opaque soot coating, Eq. (5a) applies at $X=0$

$$
\left.\frac{\mathrm{d} G_{L}}{\mathrm{~d} X}\right|_{X=0}=\frac{3 \kappa_{c L} \varepsilon_{\mathrm{so}}}{2\left(2-\varepsilon_{\mathrm{so}}\right)}\left\{G_{L}(0)-4 n^{2} t(0)^{4} F[t(0)]\right\}
$$

When the zirconia surface is clean, $q_{\mathrm{tot}}$ consists of radiation in the semitransparent spectral band combined with convection and radiative exchange at the surface in the opaque band. Using the two-flux equation following Eq. (2) for the radiative transfer in the semitransparent band, gives

$$
\begin{aligned}
& \left.\frac{\mathrm{d} G_{L}}{\mathrm{~d} X}\right|_{x=0}=3 \kappa_{c L}\left[H_{1}\left[1-t_{c}(0)\right]+\left(1-\rho_{o}\right)\left(t_{s 1}^{4}\left[1-F\left(t_{s 1}\right)\right]\right.\right. \\
& \left.\left.\quad-t_{c}(0)^{4}\left\{1-F\left[t_{c}(0)\right]\right\}\right)-\tilde{q}_{\mathrm{tot}}\right]
\end{aligned}
$$

For a clean thermal barrier coating, Eq. (5c) gives

$$
G_{L}(0)=4 \frac{1-\rho_{o}}{1-\rho_{i}} t_{s 1}^{4} F\left(t_{s 1}\right)+\left.\frac{2}{3 \kappa_{c L}} \frac{1+\rho_{i}}{1-\rho_{i}} \frac{\mathrm{d} G_{L}}{\mathrm{~d} X}\right|_{X=0}
$$

At the interface between the coating and the metal wall, Eq. (5b) applies in a similar form to Eq. (10a).

\section{Solution Method}

An iterative solution was used. Since $t(X)$ is unknown, the opaque heat conduction solution was used as a first guess for $t_{c}(X)$ and $\tilde{q}_{\text {tot }}$. When the coating surface is clean, $\mathrm{d} G_{L}(0) / \mathrm{d} X$ was found from Eq. $(10 \mathrm{~b})$, and $G_{L}(0)$ from Eq. (10c). Equation (9) for $G_{L}(X)$ was solved using Runge-Kutta numerical integration. The temperature distribution in the coating was then obtained from Eq. (8). By using the temperature $t_{c}(1)=t_{\mathrm{mh}}$ Eqs. (6) and (7) in dimensionless form were solved simultaneously for $t_{\mathrm{mc}}$ and a new value of $\tilde{q}_{\mathrm{tor}}$. The $\mathrm{d} G_{L}(1) / \mathrm{d} X$ from Eq. (5b), as in the form of Eq. (10a), was evaluated and compared with $\mathrm{d} G_{L}(1) / \mathrm{d} X$ from the Runge-Kutta solution. The new $t_{c}(X)$ and $\tilde{q}_{10}$ were adjusted by using a small fraction of the change from the previous iteration, and the adjustments 
included making $\mathrm{d} G_{L}(1) / \mathrm{d} X$ from the dimensionless form of Eq. (5b) converge toward the Runge-Kutta solution value. At convergence, agreement was obtained to at least three significant figures. The Runge-Kutta solution was checked by using 20,40 , and 80 increments; no change to at least four significant figures was obtained by using 80 increments. For typical zirconia thermal barrier coatings the absorption optical thickness is small and the scattering albedo is close to one. For these conditions the shooting method used for solving the two-point boundary value problem for $G_{L}$ converged without difficulty.

The iteration method changes somewhat when the zirconia coating has an opaque soot layer on its exposed surface. The previous results for clean opaque and semitransparent coatings were used to estimate trial $t_{c}(X)$ and $G_{L}(0)$ values. With $t_{\mathrm{so}}=$ $t_{c}(0), \tilde{q}_{10 t}$ was obtained from Eq. (1) in dimensionless form, and $\mathrm{d} G_{L}(0) / \mathrm{d} X$ from Eq. (10a). The iteration then proceeds as described for the coating with a clean surface.

\section{Results and Discussion}

Illustrative temperatures are shown in Fig. 2 for a zirconia coating on a high alloy steel combustion liner. The solid lines are for an uncoated metal wall; the lower line is for oxidized metal $\left(\varepsilon_{m}=0.6\right)$ on both sides, and for the upper line the metal is also covered with soot on the combustion side $\left(\varepsilon_{\mathrm{so}}=0.99\right)$. In both instances the temperatures are above the metal melting point, and so a thermal barrier coating must be used. A limiting calculation is for a zirconia coating assumed to be opaque (dashed lines). For a $1-m m$-thick coating the metal temperature is substantially reduced, and having soot on the coating has only a small effect as the coating thermal conductivity is mostly controlling. If the coating can be kept clean, there is a further benefit with the coating semitransparent. The high scattering of zirconia reflects away much of the incident radiation and temperatures in the zirconia are considerably decreased (lower dot-dash line), with the hot side of the metal reduced about $20 \mathrm{~K}$. If, however, the zirconia becomes covered with soot, the metal temperatures are increased. The incident radiation is absorbed by the soot on the zirconia surface, and the

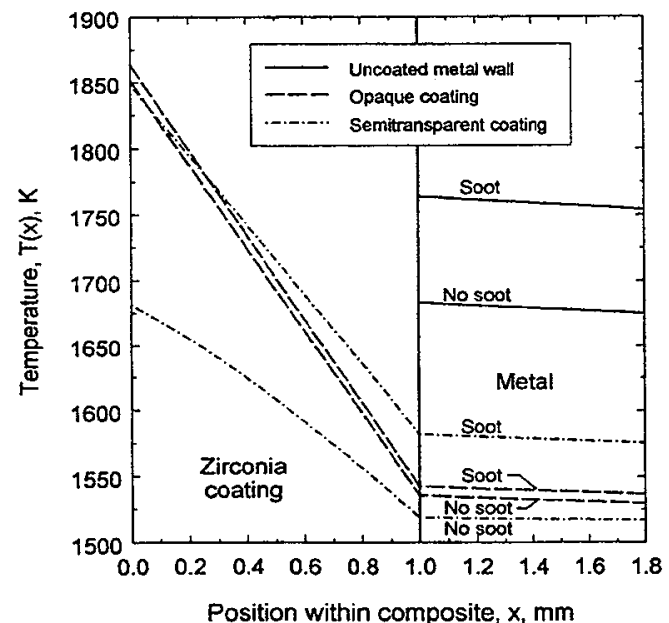

Fig. 2 Combustor liner wall temperature distributions for oxidized metal without a coating, with an opaque thermal barrier coating, and with a semitransparent thermal barrier coating with and without soot on its exposed surface. On the cooled side, radiation is to large surroundings, $\varepsilon_{x}$ in Eq. (7a). Parameters (units are in the Nomenclature): $h_{1}=250, h_{2}=110, k_{c}=0.8, k_{m}=33, \delta_{c}$ $=10^{-3}, \delta_{n}=0.794 \times 10^{-3}, n=1.58, a=30$, and $\sigma_{s}=10^{4}$ for $\lambda<$ $5 \mu \mathrm{m}, T_{s 1}=T_{p 1}=2000$, and $T_{s 2}=T_{z^{2}}=800$.

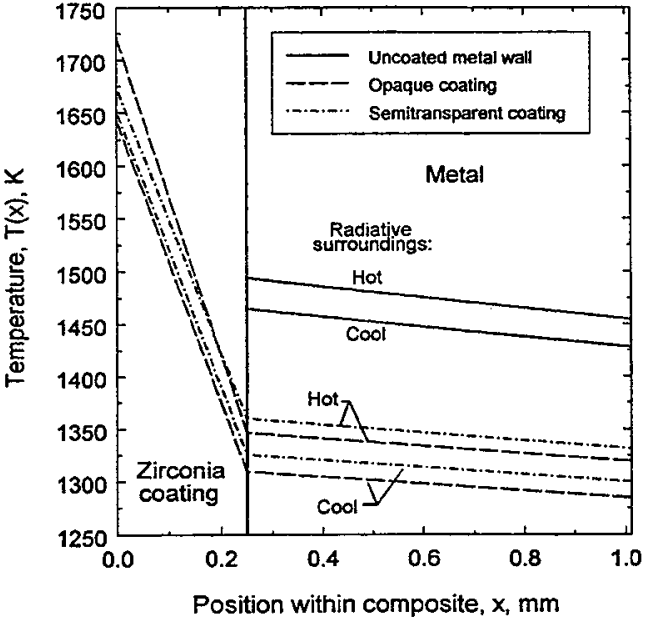

Fig. 3 Turbine blade wall temperature distributions for clean metal without a coating, with an opaque thermal barrier coating, and with a semitransparent thermal barrier coating with a clean exposed surface (no radiative loss on interior side). Parameters (units are in Nomenclature): $h_{1}=3014, h_{2}=3768, k_{c}=0.8, k_{m}=$ $33, \delta_{c}=0.25 \times 10^{-3}, \delta_{m}=0.762 \times 10^{-3}, n=1.58, a=30$, and $\sigma_{s}=10^{4}$ for $\lambda<5 \mu \mathrm{m}, T_{s 1}=T_{s 1}=2000$, and $T_{s 2}=T_{g^{2}}=1000$.

semitransparency of the zirconia acts like increased thermal conductivity; hence, an opaque coating is more effective.

Illustrative results are shown in Fig. 3 for a 0.25 -mm-thick zirconia coating on a turbine blade. The inside of the blade is assumed isothermal, and so internal radiative heat loss is omitted. Two cases are considered for the exterior:

1) The blade is surrounded by other cooled blades so that there is no external radiative heating.

2) The blade is exposed to blackbody surroundings at $T_{s 1}$ from the combustor.

The zirconia surface is assumed to be clean. Temperatures are shown for bare metal, an opaque zirconia coating, and a semitransparent zirconia coating. With or without external radiative heating, the relative positions of the $T(x)$ are the same; semitransparency yields higher metal temperatures than an opaque coating. For the higher convective heat transfer coefficients on a turbine blade compared with a combustor liner, the radiative effects are relatively too small for scattering to have sufficient benefit by blocking incident radiation for hot surroundings. Semitransparency increases the effective coating thermal conductivity for heating primarily by convection, and metal temperatures are increased. These results illustrate how the analysis can be used to examine the effect of semitransparency for various parameters; semitransparency is found to be beneficial for limited conditions.

\section{References}

'Wahiduzzaman, S., and Morel, T.. "Effect of Translucence of Engineering Ceramics on Heat Transfer in Diesel Engines," Oak Ridge National Lab., ORNL/Sub/88-22042/2, Oak Ridge, TN, April 1992.

${ }^{2}$ Spuckler, C. M., and Siegel, R., "Two-Flux and Diffusion Methods for Radiative Transfer in Composite Layers," Journal of Heat Transfer, Vol. 118, No. 1, 1996, pp. 218-222.

${ }^{3}$ Siegel, R., and Spuckler, C. M., "Approximate Solution Methods for Spectral Radiative Transfer in High Refractive Index Layers," International Journal of Heat and Mass Transfer, Vol. 37. Suppl. 1, 1994, pp. 403-413.

${ }^{4}$ Siegel, R., and Howell, J. R., Thermal Radiation Heat Transfer, 3rd ed., Hemisphere, Washington DC, 1992, pp. 23, 33.

${ }^{5}$ Makino, T., Kunitomo, T., Sakai, I., and Kinoshita, H., "Thermal Radiative Properties of Ceramic Materials," Transactions of the Japan Society of Mechanical Engineers, Vol. 50, No. 452, 1984, pp. $1045-1052$. 